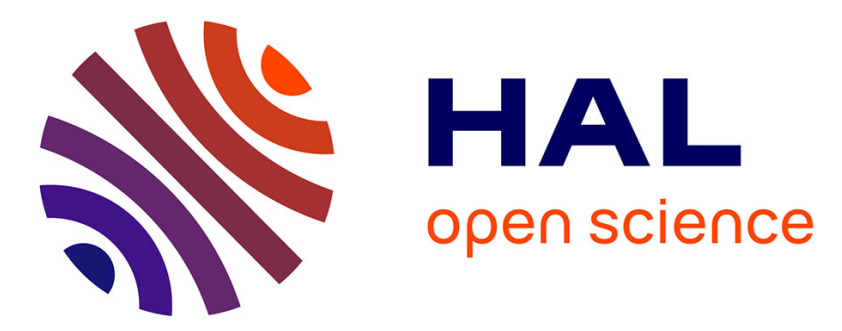

\title{
Numerical modeling of vacuum-assisted resin transfer molding using multilayer approach
}

Raounak Loudad, Abdelghani Saouab, Pierre Beauchene, Romain Agogue, Bertrand Desjoyeaux

\section{To cite this version:}

Raounak Loudad, Abdelghani Saouab, Pierre Beauchene, Romain Agogue, Bertrand Desjoyeaux. Numerical modeling of vacuum-assisted resin transfer molding using multilayer approach. Journal of Composite Materials, 2017, 51 (24), pp.3441-3452. 10.1177/0021998316687145 . hal-01558436

\section{HAL Id: hal-01558436 \\ https://hal.science/hal-01558436}

Submitted on 4 Oct 2021

HAL is a multi-disciplinary open access archive for the deposit and dissemination of scientific research documents, whether they are published or not. The documents may come from teaching and research institutions in France or abroad, or from public or private research centers.
L'archive ouverte pluridisciplinaire HAL, est destinée au dépôt et à la diffusion de documents scientifiques de niveau recherche, publiés ou non, émanant des établissements d'enseignement et de recherche français ou étrangers, des laboratoires publics ou privés. 


\title{
Numerical modeling of vacuum-assisted resin transfer molding using multilayer approach
}

\author{
Raounak Loudad', Abdelghani Saouab', Pierre Beauchene', \\ Romain Agogue $^{2}$ and Bertrand Desjoyeaux ${ }^{3}$
}

\begin{abstract}
Vacuum-assisted resin transfer molding (VARTM) is a very suitable solution for composite manufacturing industry. It allows the manufacturing of large and complex shape parts at low costs. However, the simulation of this process is complicated due to myriad physical phenomena involved, specifically the strong coupling between the resin flow and the preform compressibility, i.e. hydro-mechanical coupling. Moreover, the use of the distribution medium involves two types of flow: Planar flow and through-the-thickness flow. These flows cannot be considered together by a 2D model. On the other hand, 3D models require an important amount of computation time. This article presents a VARTM modeling approach that takes into account the hydro-mechanical coupling and the coexistence of planar and transverse flows. The proposed modeling approach allows the simulation of the infusion process in the case of multilayer preform with different materials and orientations, including the distribution medium. This model is validated experimentally based on several infusions.
\end{abstract}

\section{Keywords}

Polymer-matrix composites, finite element analysis, vacuum-assisted resin transfer molding, process simulation, permeability

\section{Introduction}

Vacuum-assisted resin transfer molding (VARTM) is a closed mold process that allows the manufacturing of high-performance composite parts. It is also known as vacuum infusion (VI), ${ }^{1}$ vacuum bag resin transfer molding (VBRTM), ${ }^{2}$ resin injection under flexible tooling (RIFT), ${ }^{3}$ Seemann composites resin infusion molding process (SCRIMP $\left.{ }^{\mathrm{TM}}\right)^{4}$ and liquid resin infusion (LRI). ${ }^{5}$ This process consists in placing the preform fabric between a half rigid mold and a vacuum bag. Then, a vacuum is generated inside the mold, making the vacuum bag compact to the preform and the resin infuses into the preform. This solution is very cost efficient, particularly for large parts with complex shapes. However, its understanding is still very limited. In fact, the flexibility of the vacuum bag makes the part thickness vary depending on the resin pressure. The variation in the part thickness, and thereby in its fiber volume fraction, results in changes in the preform permeability, and varies the resin pressure. This mutual influence, between the resin flow and the preform deformation, is called the hydro-mechanical (HM) coupling.

In order to ensure the mold filling in the case of large parts, a distribution medium (DM) of very high permeability is placed between the vacuum bag and the preform. The use of the DM accelerates the preform impregnation by adding the filling through the thickness direction, also called transverse flow.

The modeling of the infusion process has been widely studied in the literature. Several analytical

\footnotetext{
'Laboratoire Ondes et Milieux Complexes, Normandie Université, France

${ }^{2}$ Département Matériaux et Systèmes Composites, Office National d'Etudes et de Recherches Aérospatiales (ONERA), France

${ }^{3}$ SAFRAN Nacelles, France
}

\section{Corresponding author:}

Abdelghani Saouab, Laboratoire Ondes et Milieux Complexes, UMR CNRS 6294, LABEX EMC3, Normandie Université, 53, rue de Prony, CS 80540, 76058 Le Havre Cedex, France.

Email: abdelghani.saouab@univ-lehavre.fr 
and numerical methods have been developed for this purpose.

The analytical methods have been used to study the infusion in simple unidirectional cases. These studies are divided into two categories: on one hand, the study of HM coupling in infusion process without the use of the DM, ${ }^{6-8}$ and on the other hand, the study of flow front kinetics in infusion processes using the DM by simplifying the HM coupling model..$^{9-12}$

Several numerical approaches have been used to study infusion processes. Many authors have developed models based on finite difference method. ${ }^{13,14}$ Kessels et al. ${ }^{15}$ have modeled RIFT process in $2.5 \mathrm{D}$ using finite volume method. Gantois et al. ${ }^{16}$ have developed a modeling approach based on boundary element method. Rouhi et al. ${ }^{17,18}$ proposed a dual-scale HM coupling of the infusion process. Sirtautas et al. ${ }^{19}$ developed a mesoscopic model for draping and infusion simulation. Poorzeinolabedin et al. ${ }^{20}$ modeled RIFT process using 2.5D PAMRTM code without considering the HM coupling.

Celle et al. ${ }^{5}$ and Dereims et al. ${ }^{21}$ have proposed a non-isothermal finite element model of infusion in which the flow is governed by Stokes law in the DM and by Darcy law in the preform. These models are based on a mixed formulation using $\mathrm{P} 1+/ \mathrm{P} 1$ elements and Arbitrary Lagrangian Eulerian (ALE) formulation.

Control volume finite element method (CVFEM) has been widely used in the literature to simulate RTM and infusion processes. Kang et al. ${ }^{2}$ have developed a diphasic CVFEM model of resin infusion in $3 \mathrm{D}$, which allows to simulate the creation of dry zones. Dong ${ }^{22}$ has proposed an adaptation of the 3D CVFEM modeling called "Equivalent medium method". This adaptation consists in increasing the thickness of the DM so as to mesh the structure conveniently.

In order to reduce the computation time of 3D modeling, some authors (Calado and Advani, ${ }^{23}$ Chen et al. ${ }^{24}$ and Yoon and Dolan ${ }^{25}$ ) have modeled the resin flow in $2 \mathrm{D}$ by using an equivalent permeability that takes into account the flow front shape. These models have been validated by comparing $3 \mathrm{D}$ models results. Simancek and Advani ${ }^{26}$ proposed to homogenize the DM and the preform permeability and introduced a correcting factor so as to have the same front advance. Han et al. ${ }^{4}$ and Kang and $\mathrm{Lee}^{27}$ have modeled SCRIMP using a hybrid 2.5D-3D CVFEM model.

In sum, since the infused parts have usually shell-like shapes, it is suitable to model them in 2D. However, the coexistence of the planar flow and the transverse flow, involved by the DM, makes the flow happen in $3 \mathrm{D}$. This leads to the modeling dimensions dilemma between simplified 2D modeling and time consuming $3 \mathrm{D}$ modeling. In the case of $2 \mathrm{D}$ modeling, the transverse flow cannot be considered and the superposed layers with different materials and orientations are homogenized into one. ${ }^{23-26}$ Whereas in 3D modeling, a fine mesh is required to guarantee convenient shape factor elements, since the part thickness is very small comparing to the other directions $s^{2,4,22,28}$ which increases the computational time.

Another alternative has been introduced by Young ${ }^{29}$ who has developed a two-layer model, one modeling the DM and the other the preform. This model was validated analytically based on simplifying assumptions such as considering constant permeability or equal constant porosity in both layers.

In this paper, we propose a model of mold filling by VARTM that takes into account the HM coupling with both planar and transverse flows. This model is based on a multilayer approach that is less time consuming than 3D modeling.

\section{Governing equations: HM coupling}

The fluid flow through deformable fibrous media has been studied by many authors. ${ }^{9-12,30}$ In this study, we use the model proposed by Kempner and Hahn. ${ }^{31}$ This model is based on Darcy equation ${ }^{32}$ and the mass conservation equation in a deformable elementary representative volume (ERV). The combination of these equations leads to the following governing equation of the resin pressure $P$

$$
\nabla\left(\frac{-K_{i j}}{\mu} \nabla P\right)=\frac{1}{V_{f}} \frac{\partial V_{f}}{\partial t}+\frac{1}{V_{f}} u_{s} \frac{\partial V_{f}}{\partial t}
$$

where $V_{f}$ is fiber volume fraction, $K_{i j}$ the medium permeability, $\mu$ the viscosity, and $u_{s}$ the solid velocity.

The second term in the right hand equation is negligible because the fiber velocity is negligible comparing to fluid velocity. ${ }^{6}$ The governing equation of the pressure is then simplified

$$
\nabla\left(\frac{-K_{i j}}{\mu} \nabla P\right)=\frac{1}{V_{f}} \frac{\partial V_{f}}{\partial t}
$$

The force equilibrium in the porous medium leads to the relationship between the resin pressure $P$ and the preform compressibility $\sigma$. This relationship is given by the theorem of Terzaghi ${ }^{33}$

$$
\sigma=P_{a t m}-P
$$

where $P_{a t m}$ is the atmospheric pressure.

The flow modeling requires the behavior laws of the fibrous medium in terms of permeability and compressibility. These physical quantities evolve during the infusion. In fact, the fiber volume fraction $V_{f}$ is 
expressed in terms of compressibility

$$
V_{f}=F_{c}(\sigma)
$$

whereas the permeability varies as a function of the fiber volume fraction

$$
K_{i j}=F_{p}\left(V_{f}\right)
$$

The HM coupling represents the relationship between the fluid flow and the fibrous medium deformation. This coupling is modeled by the equation system (2) to (5) combined with the boundary conditions which are the imposed pressures at the injection and vent gates.

\section{Numerical resolution scheme}

The HM equation system is solved numerically using the CVFEM. ${ }^{34}$ This method, based on the Eulerian approach, uses a fixed mesh which saves the remeshing computation time. In fact, instead of moving the meshed domain after each time step, this domain evolves by changing the filling factor of the control volumes using the volume of fluid (VOF) method (Figure 1(a)). However, although this method is fast, it does not ensure the accurate application of the boundary conditions at the flow front. Actually, the flow front position is estimated by interpolation between nodes, whereas the boundary conditions are applied on the nodes. To address this problem, a mesh refining method at the flow front region called floating imaginary nodes and elements (FINE) ${ }^{34}$ is used in this study. This method consists in dividing the partially filled elements into completely filled and empty sub-elements.

In order to model the infusion of stack of different materials and orientations, we model the HM coupling in each layer with a surface mesh that includes a degree of freedom on the thickness. The transverse flow is modeled by $1 \mathrm{D}$ elements that link the surface mesh as shown in Figure 1(b).

As in the conventional CVFEM, the mass balance is rigorously respected within the control volume by integrating fluxes on the control volume borders. To do so, the divergent theorem is applied on the pressure governing equation (2)

$$
\int_{C S}\left(\frac{-\overline{\bar{K}}}{\mu} \overline{\nabla P}\right) \cdot \bar{n} \mathrm{~d} S=\int_{C V} \frac{1}{V_{f}} \frac{\partial V_{f}}{\partial P} \frac{\partial P}{\partial t} \mathrm{~d} V
$$

Using the linear interpolation of the elements, the pressure gradient can be expressed in terms of nodal pressures and the nodes coordinates. ${ }^{34}$ The source term is integrated on the control volume and expressed in terms of the present nodal pressure, the nodal pressure at the previous time step and the time step which corresponds to an implicit scheme.

(a)
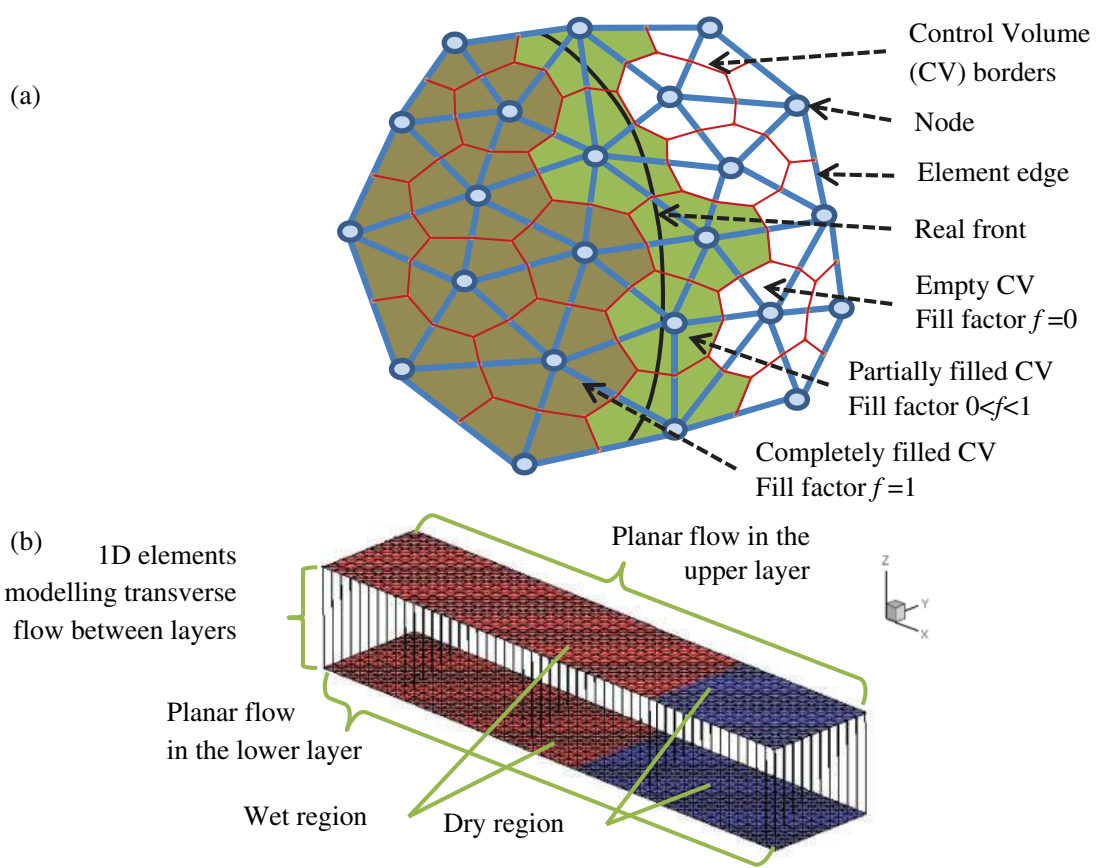

Figure I. Control volume finite element method (CVFEM) computation field in (a) the conventional 2D approach and in (b) the multilayer approach. 
And finally, the system matrix is constructed and resolved after applying the boundary conditions.

In the multilayer modeling approach, the system global matrix contains less than half of the non-null coefficients of the system global matrix in the 3D modeling. Moreover, 3D modeling generates an error due to the homogenization of properties, such as the porosity, in the nodes shared by two layers, which requires special treatment in the flow front or mesh refinement. All these considerations makes the computation time in the multilayer approach less important than in $3 \mathrm{D}$ modeling.

\section{Experimental characterization}

In order to validate experimentally the proposed model of VARTM, we characterized experimentally the compressibility and the permeability behavior laws for both reinforcement material and DM. These behavior laws were used to simulate the experimental infusions that we performed using these materials.

\section{Compressibility}

Preform. Before the beginning of each infusion, we subjected the infused stack to three pre-compaction cycles in order to increase the fiber volume fraction of the preform. These cycles were performed under the flexible bag using the vacuum. Figure 2 shows the total thickness evolution over time in the case of plate infusion described in "Experimental infusion recording" section.

This total thickness of four reinforcement layer and $\mathrm{DM}$ is measured on a point located at $29 \mathrm{~cm}$ from the injection line.

The preform has a viscoelastic behavior and a plastic behavior related to the nesting of the layers. Also, this behavior depends on whether the preform is dry or wet. In this study, we modeled the compressibility behavior law with a nonlinear elastic model. Hence, in order to

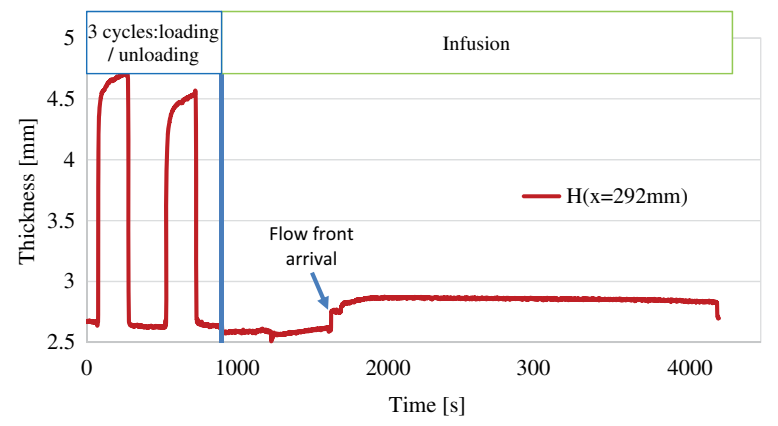

Figure 2. The evolution of the infused stack thickness at $29 \mathrm{~cm}$ from the injection line during pre-compaction cycles and infusion-plate infusion case. characterize the closest behavior law to the preform compressibility during the infusion, we performed the same protocol of compressibility using the universal testing machine. Figure 3 presents the experimental thickness and compressibility versus time applied on a sample of four layers of the reinforcement used in this study.

From Figure 3, we notice the existence of plastic strain caused by the layers nesting. Relaxation phenomena are also observed from the asymptotic evolution of the thickness while the compressibility remains constant. In addition, when the thickness is expressed in terms of compressibility, we notice hysteresis behavior from the difference between loading and unloading curves. These relaxation behavior and hysteresis behavior prove that the preform has a viscoelastic behavior. Moreover, the compressibility behavior differs depending on whether the preform is dry or wet.

After the pre-compaction cycles, the compressibility is measured during the unloading of the sample as during the infusion, where the preform compressibility decreases as a result of the increase of the fluid pressure. This measured compressibility is assumed to be representative of the fabric behavior during infusion, although the preform is unloaded with the different velocities depending on the distance from the injection.

This compressibility is modeled by Grimsley model ${ }^{35}$ expressed in equation (7)

$$
H=\frac{A \sigma+B}{C \sigma+1}
$$

where $\quad \mathrm{A}=7.1210^{-7} \mathrm{~m} \cdot \mathrm{Pa}^{-1} ; \quad \mathrm{B}=3.03 \mathrm{~mm} ; \mathrm{C}=$ $3.7710^{-4} \mathrm{~Pa}^{-1}$ and $H$ the thickness of the four layers sample. The relationship between the preform thickness $H$ and its fiber volume fraction $V_{f}$ is given by the following equation ${ }^{35}$

$$
H \cdot V_{f}=H_{i n i} \cdot V_{f_{i n i}}
$$

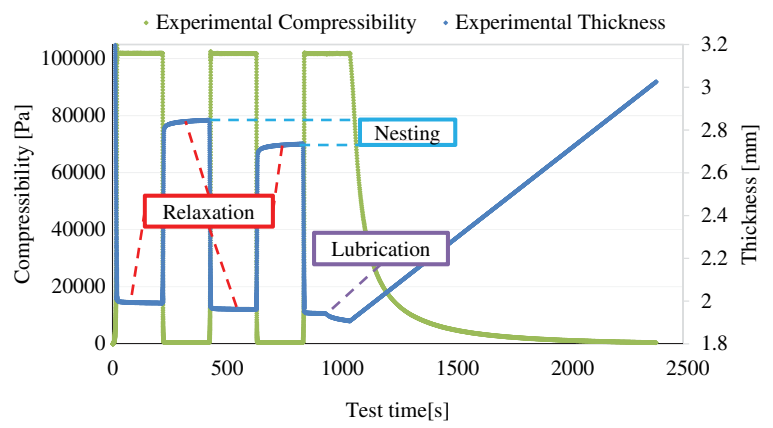

Figure 3. Four layers preform compressibility and thickness following the infusion protocol. 
where $H_{i n i}$ and $V_{f_{i n i}}$ are, respectively, the initial preform thickness and initial fiber volume fraction. The Grimsley model is very suitable to infusion process because, unlike power model, it can fit low compressibility values accurately. Figure 4(a) shows that this model fits perfectly the experimental data in the infusion application field of compressibility.
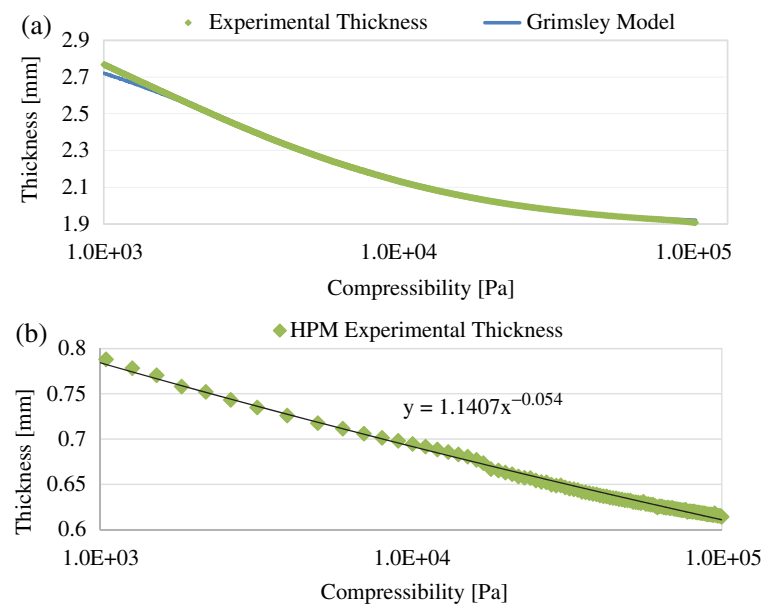

Figure 4. Experimental compressibility behavior law of four layers preform (a) and the distribution medium (b).
Distribution medium. The DM compressibility has also been measured during the unloading. It is modeled by a power model with high accuracy as shown in Figure 4(b).

\section{Permeability}

The permeability is measured by applying air flow rates through the sample and extracting the pressure at several positions. This experiment is repeated for many fiber volume fractions in the compressibility application field. The use of air to measure the permeability has been widely used in the literature, ${ }^{36-40}$ and it requires vigilance to not exceed the limit air velocity so as the Reynolds number condition for laminar flow stays respected. Scholz et al. ${ }^{36}$ has proposed a correcting factor related to air compressibility. In our measures this factor is always close to one.

Planar permeability. The planar permeability bench contains four pressure sensors at the horizontal and vertical directions and three on the diagonal direction as shown in Figure 5(a). The air flows from the central injection point to the vent located at the borders.

For a specific fiber volume fraction value, the experimental pressure field is measured. Then, from this
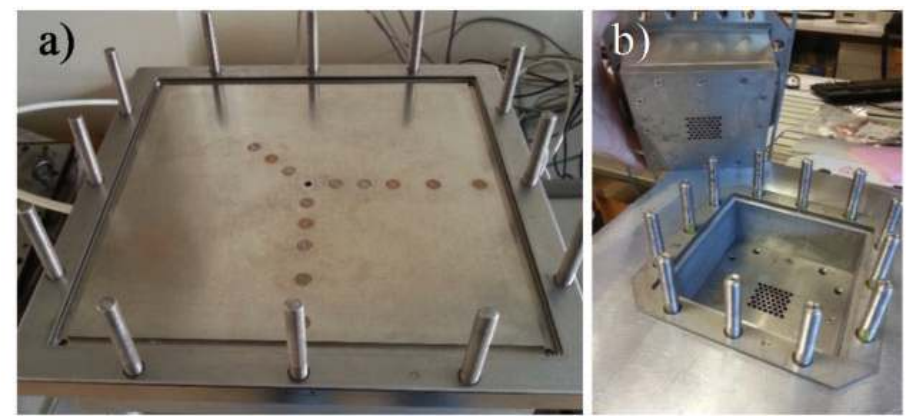

c) Upper flow front camera
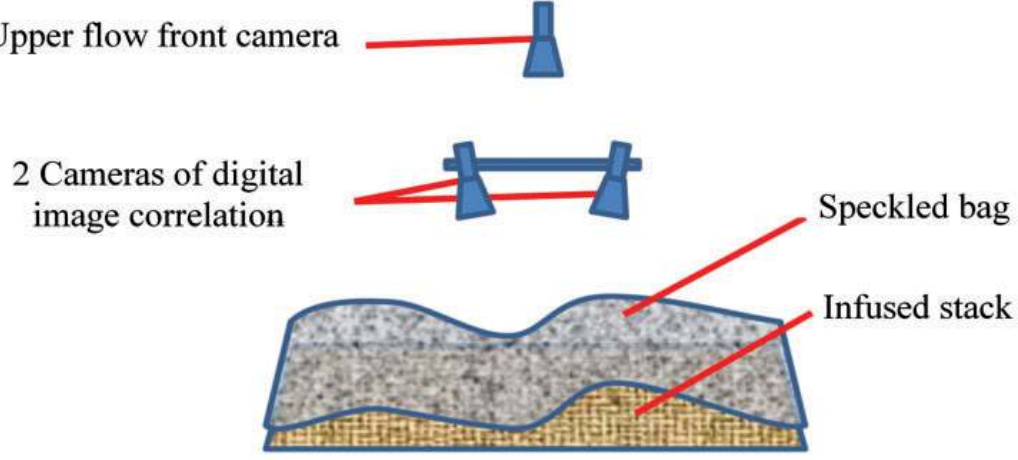

Lower flow front camera

Figure 5. Experimental device (a) planar permeability bench, (b) transverse permeability bench, and (c) infusion recording device (front tracking and DIC). 
pressure field and the applied air flow rate at the injection point, a reverse analysis using $\mathrm{COMSOL}^{\mathrm{TM}}$ allows the identification of the planar permeability tensor coefficients, which are the permeability in both principal directions and the orientation angle. ${ }^{41}$

Figure 6 presents the preform planar permeability in the principal directions $K_{1}$ and $K_{2}$ versus the fiber volume fraction. These behavior laws are modeled by a power model.

Due to its low thickness of the DM and its high permeability, the planar permeability of the DM is highly influenced by the border effects. In this study, a constant value has been identified for the DM planar permeability in the flow direction by a reverse analysis on the infusions performed experimentally

$$
K_{\mathrm{DM} 1}=1.610^{-9} \mathrm{~m}^{2}
$$

Transverse permeability. The transverse permeability is identified by injecting air through the thickness direction of the sample using the bench shown in Figure 5(b). In this bench, a pressure sensor is placed at the injection side, and the vent at the atmospheric pressure is located at the other side of the sample.

The transverse permeability $K_{3}$ is deduced from the injection relative pressure, the sample's thickness, the air flow rate and its viscosity using Darcy law. The preform transverse permeability versus the fiber volume fraction experimental curve is given in Figure 6. This behavior law is modeled by a power law with reasonable accuracy.

The DM transverse permeability is very high in comparison with the preform one. Hence, its influence on the flow is negligible as shown in equation (9) ${ }^{12}$

$$
\frac{h_{d}+h_{p}}{k_{T}^{t}}=\frac{h_{d}}{k_{d}^{t}}+\frac{h_{p}}{k_{p}^{t}} \cong \frac{h_{p}}{k_{p}^{t}}
$$

where $h_{p}$ and $h_{d}$ mean the thickness of the preform and the DM, $k_{p}^{t}$ and $k_{d}^{t}$ are respectively their transverse permeability and $k_{T}^{t}$ their equivalent transverse permeability.

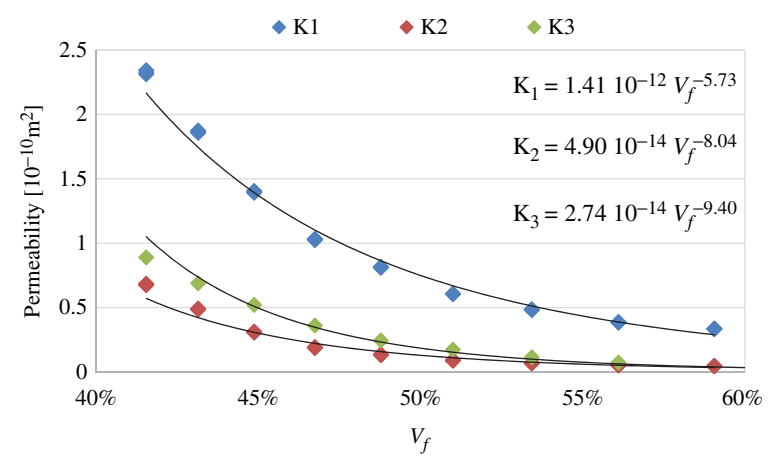

Figure 6. Preform permeability in the principal directions.
In this study, we took a DM transverse permeability value of $10^{-6} \mathrm{~m}^{2}$ so as to neglect its influence.

\section{Experimental infusion recording}

In order to validate the proposed model experimentally, two infusions were recorded and simulated. The recorded experimental data are the DM and the total thickness of the stack constituted of the preform and the DM and the flow front positions in the preform.

The thickness of the stack is measured by digital image correlation (DIC). ${ }^{42}$ This method consists in filming the vacuum bag from two different angles of view and deducing the distribution of its height. The flow front is tracked on both sides of the mold using two cameras positioned over and below the infusion bench as illustrated in Figure 5(c).

\section{Experimental validations}

\section{First case: Plate infusion}

First case data. This infusion case is performed using a silicone oil of 1 Pa.s viscosity. The infused stack is composed of four layers of the characterized preform and the characterized DM. The length of these layers is $50 \mathrm{~cm}$ and the injection pressure is $0.9610^{5} \mathrm{~Pa}$, as the oil free surface was $30 \mathrm{~cm}$ under the mold (Figure 7(a)).

This infusion was simulated using the mesh presented in Figure 7(b). The upper surface mesh models the DM and the lower surface mesh models the fourlayer preform.

\section{Results comparison}

Thickness. Figure 8 presents the experimental and numerical thickness of the infused stack at the mold

(a)

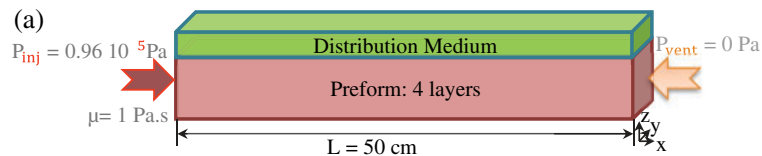

(b)

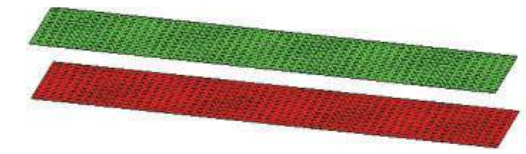

(c)

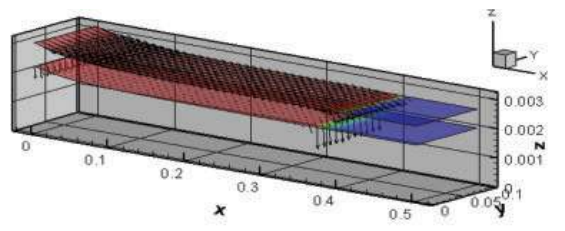

Figure 7. Plate infusion case study: (a) case data, (b) numerical mesh and (c) numerical velocity vectors during infusion. 
filling time, with the $\mathrm{x}$-axis representing the position in the infused plate. The experimental results are limited to $318 \mathrm{~mm}$, which is the DIC camera field of vision. Increasing fluctuations in the experimental thickness

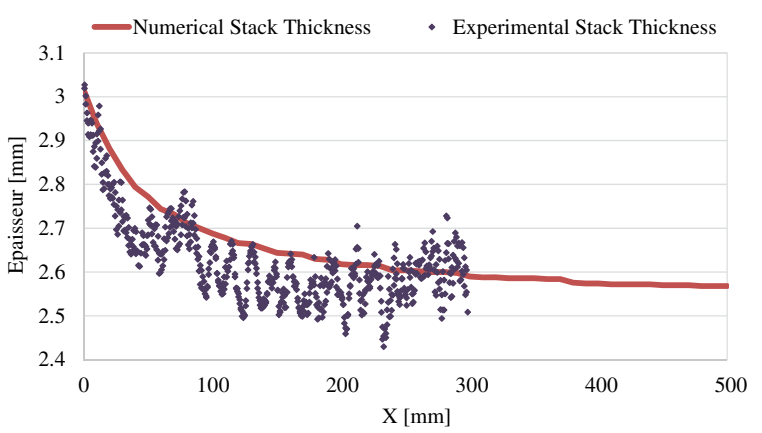

Figure 8. Total thickness distribution at the mold filling in the plate infusion case.

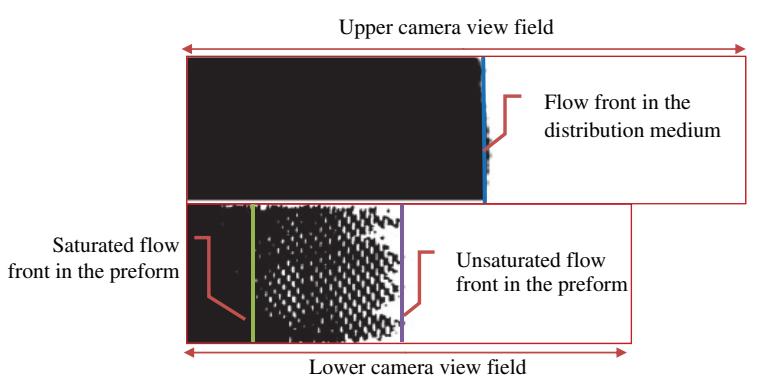

Figure 9. Processed images of the infusion on both sides. according to the position are observed in Figure 8 . These fluctuations are due to the collapse of the vacuum bag into the DM under the difference between the fluid and external pressure. Hence, the numerical thickness corresponds to the maxima of these corrugations. Figure 8 shows a reasonable match between these results.

Flow front kinetics. The recorded pictures of the flow front are processed by Image J. ${ }^{43}$ As shown in Figure 9, the flow front position in the DM can be easily identified thanks to the discontinuity between the wet and dry region. On the other hand, an unsaturated region exists in the preform between the saturated region and the dry region. This is caused mainly by the double scale porosity of the preform. Thus, one flow front position in the DM is tracked whereas both saturated and unsaturated flow front positions were recorded in the preform.

Since the numerical model does not consider the double scale porosity, only one numerical flow front is modeled in the preform. Figure 10(a) shows the numerical and experimental flow front kinetics on both sides of the mold. Again, the experimental results are limited to the field of vision of the cameras.

Figure 10(a) shows that the numerical front position in the DM side is close to the experimental results. This is due mainly to the fact that the DM permeability has been determined by reverse analysis. On the preform side, the numerical front agrees more with the experimental unsaturated front than the saturated one.
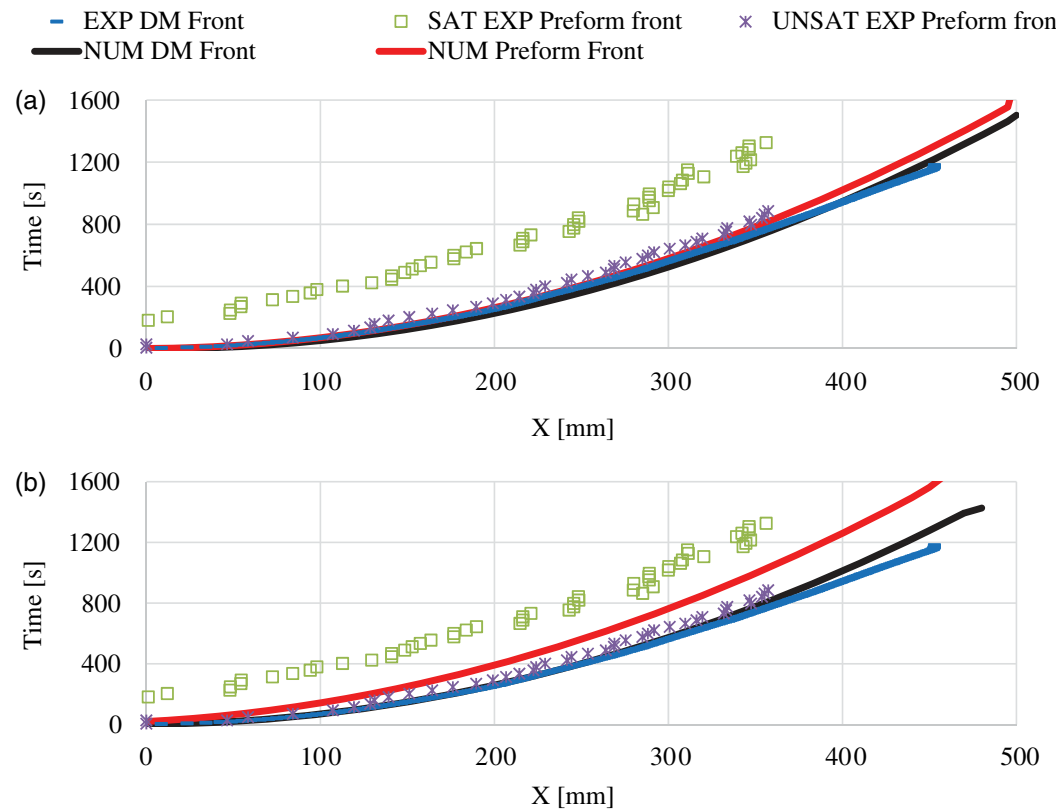

Figure 10. Numerical and experimental flow front kinetics in the plate infusion case using the measurable preform transverse permeability (a) and a lower one (b). 
This is because of the measured transverse permeability of the preform that is closer to the macropores permeability than to the micropores permeability. ${ }^{44}$ In fact, since these pores are in a parallel configuration regarding through-the-thickness flow, the average permeability is an arithmetic average that is dominated by the higher permeability, ${ }^{45}$ in this case the macropores permeability.

If we use a lower transverse permeability of the preform, the gap between the flow front position in
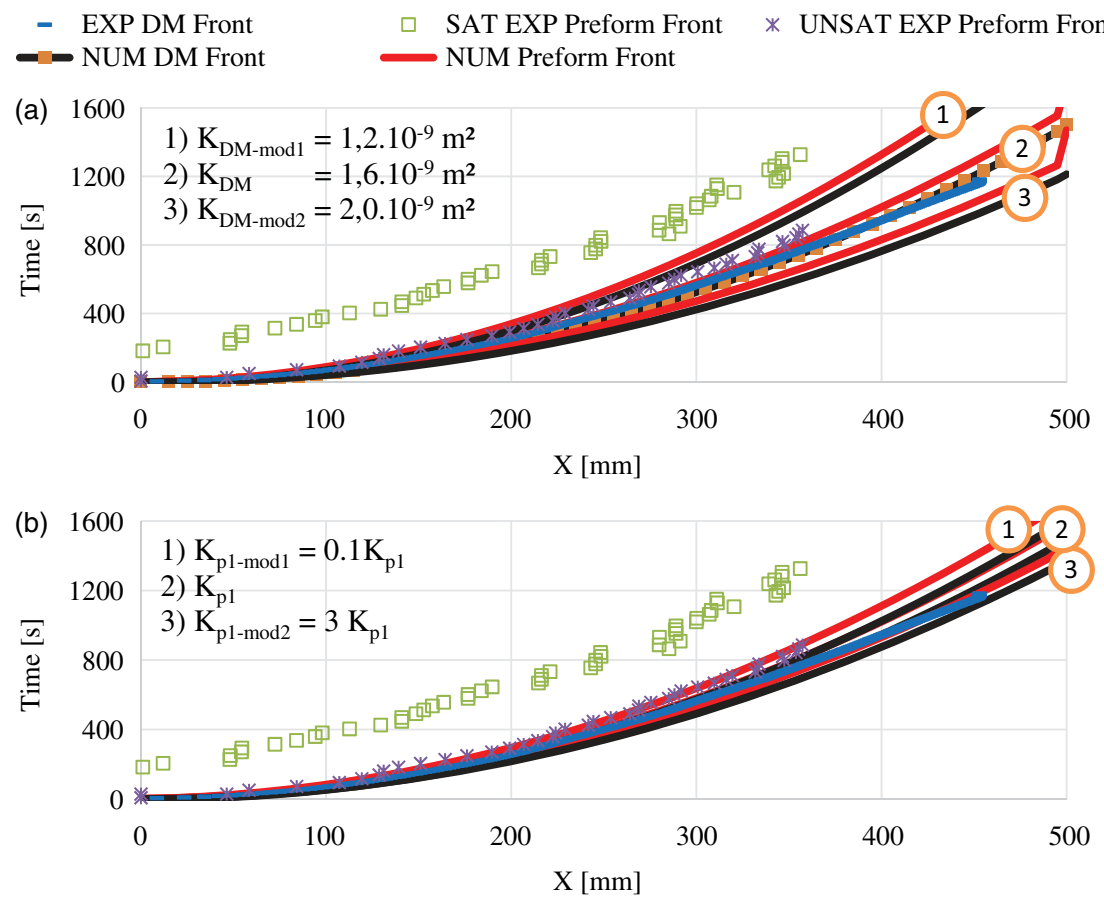

Figure II. Sensitivity analysis of the flow front kinetics on the planar permeability of the DM (a) and the preform (b) in the case of plate infusion.

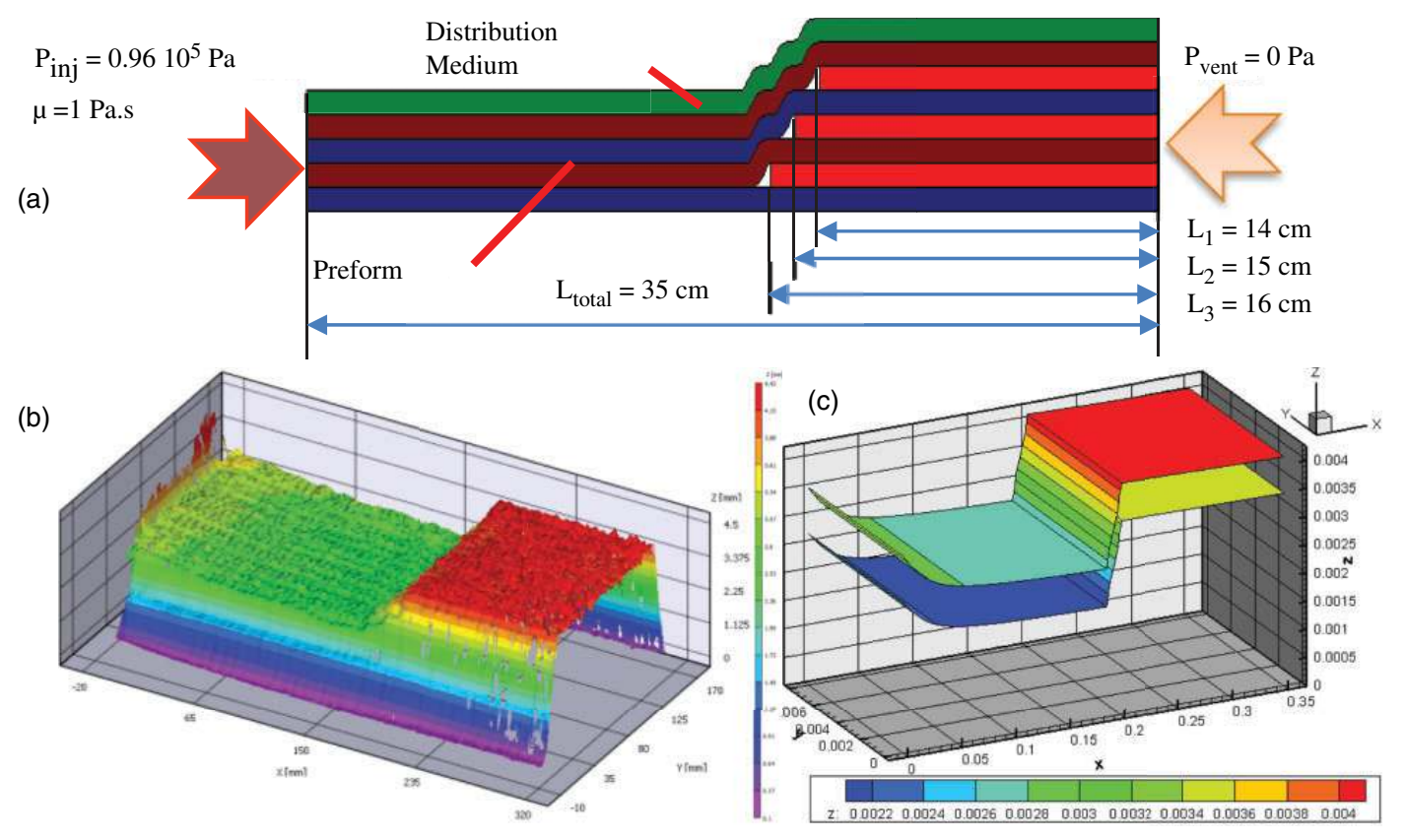

Figure 12. Ply drop case study: (a) case data, (b) ply drop shape by DIC and (c) ply drop shape by simulation. 
the DM and the preform increases. Figure 10(b) presents the numerical flow front kinetics using a modified preform transverse permeability with a correcting factor of 0.1

$$
K_{3 \text { modified }}=2.74 \times 10^{-15} V_{f}^{-9.4}
$$

Whereas the measured permeability was (Figure 10(b))

$$
K_{3 \text { measured }}=2.74 \times 10^{-14} V_{f}^{-9.4}
$$

Figure 11(a) presents the numerical flow front kinetics in three cases where the DM planar permeability is slightly modified.

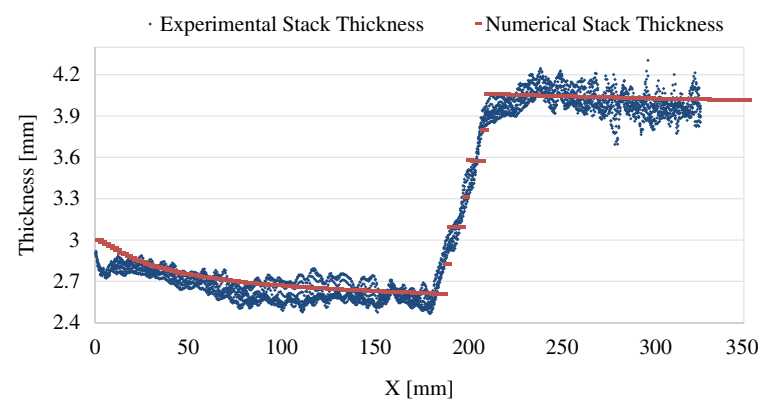

Figure 13. Total thickness distribution at the mold filling in the ply drop infusion case.
Figure 11(b) presents the numerical flow front kinetics in three cases where the preform planar permeability is considerably modified. From this sensitivity study, we notice that the infusion is highly sensitive to the DM planar permeability and lowly sensitive to the preform planar permeability. This could be explained by the fact that the DM and the preform are parallel to the flow (Figure 7(c)), which means that the flow is dominated by the higher permeability. ${ }^{45}$ The transverse permeability of the preform has an important influence on the gap between the flow front positions in the DM and the preform.

\section{Second case: Ply drop infusion}

Second case data. We study, in this case, an infusion of a ply drop from 7 to 4 plies of the characterized preform. As in the previous case, the infusion is performed using the silicone oil of $1 \mathrm{~Pa} . \mathrm{s}$ viscosity and the injection pressure is also equal to $0.96 \times 10^{5} \mathrm{~Pa}$. The data of this studied case are given in Figure 12(a).

\section{Results comparing}

Thickness. Figure 12(b) presents the thickness distribution of the infused stack measured experimentally by DIC. These values were measured at the mold
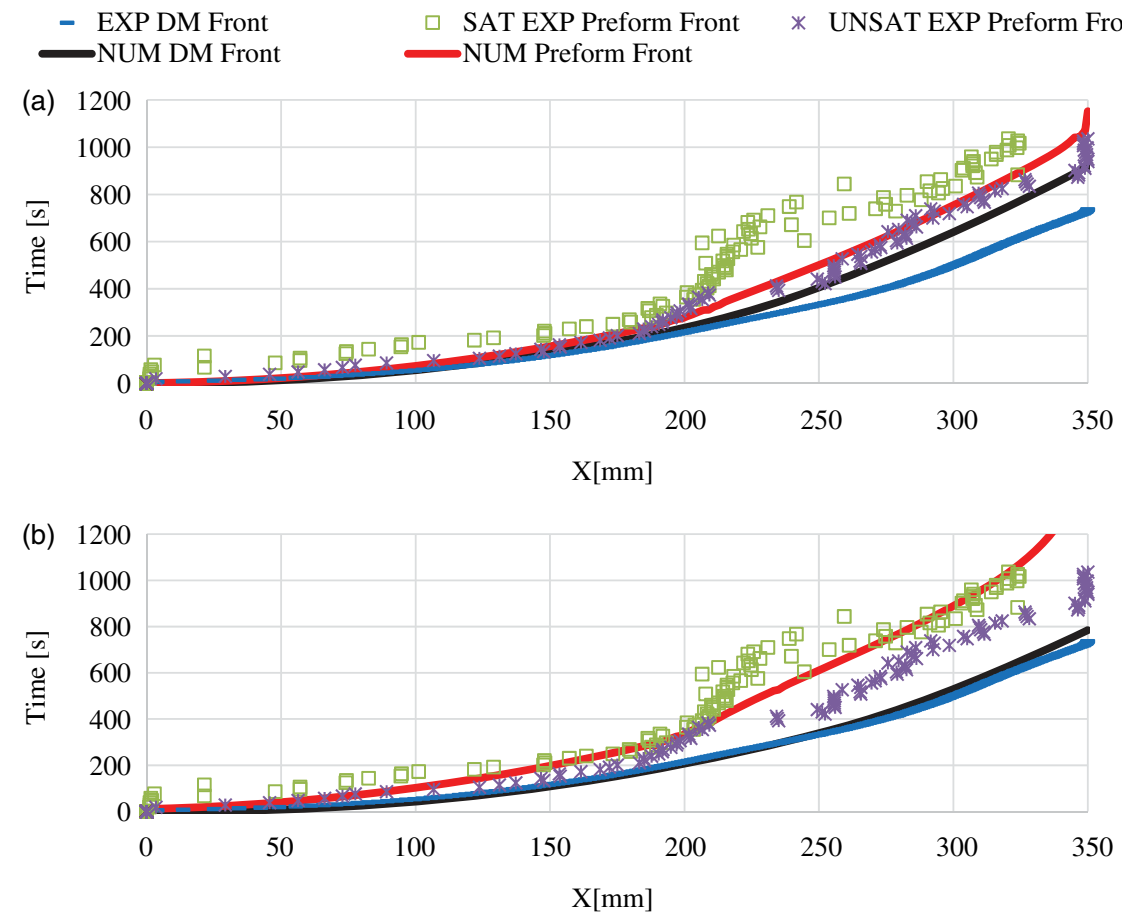

Figure 14. Numerical and experimental flow front kinetics in the ply drop infusion case using the measurable preform transverse permeability (a) and a lower one (b). 
filling time. The DM corrugation can be noted in this figure, which explains the variability of the experimental data in Figure 13.

In Figure 13, values covering one corrugation pitch were extracted. A reasonable match is observed between the experimental and the numerical results. The maximal difference between the numerical curve and the experimental fluctuations peaks is about $0.1 \mathrm{~mm}$, which represents an error of $3 \%$.

Flow front kinetics. The numerical and experimental flow front kinetics on the DM side are given in Figure 14(a). In this figure, a good match is observed between the numerical and experimental results until $200 \mathrm{~mm}$, which is the end of the plate area of four preform layers and DM. Then, in the DM, the experimental flow front becomes faster than the numerical one until $290 \mathrm{~mm}$, after that, the experimental and the numerical flow fronts evolve with a similar velocity and a constant gap of $26 \mathrm{~mm}$.

As in the previous case, we performed a simulation using a modified transverse permeability of the preform with a correction factor of 0.1 and we obtained numerical flow front kinetics closer to the experimental results (Figure 14(b)).

Again, this leads us to think that the measured transverse permeability using air flow, which represents the saturated permeability, is higher than the unsaturated permeability that we have during the infusion. In fact, the measured permeability is dominated by the permeability of the macropores because the micropores and the macropores are parallel to the flow during the measure. ${ }^{45}$ Whereas during the infusion, the macropores are filled in first place, and then the micropores are filled. During the filling of the micropores, the micropores and the macropores are in series. Hence the transverse permeability in this case is dominated by the lower permeability, ${ }^{45}$ i.e. the transverse permeability of the tows.

\section{Conclusion}

In this article, we presented a numerical modeling of the infusion process in the case multilayer preform. The proposed model takes into account the HM coupling and the flow through the thickness direction. This model, based on the proposed multilayer approach, saves a significant amount of computation time compared to $3 \mathrm{D}$ modeling.

The developed model is validated experimentally using measured behavior laws and gives satisfying results.

The sensitivity study carried out on the permeability values shows that the DM planar permeability plays the most preponderant role in determining the flow front velocity. Whereas the transverse permeability of the preform determines the gap between the flow front positions in the DM and in the preform. This transverse permeability was measured using a saturated flow, which makes it equivalent to macropores permeability. Hence, it does not correspond to the transverse filling where the macropores are filled in first place and then the micropores, making the filling of these pores in series. The flow in this case is controlled by micropores permeability. Based on this conclusion, dual scale porosity of the preform must be considered for a more accurate transverse permeability.

\section{Acknowledgment}

The collaboration with SAFRAN Nacelles is gratefully acknowledged.

\section{Declaration of Conflicting Interests}

The author(s) declared no potential conflicts of interest with respect to the research, authorship, and/or publication of this article.

\section{Funding}

The author(s) disclosed receipt of the following financial support for the research, authorship, and/or publication of this article: the PRC Composites, French research project funded by DGAC (French Civil Aviation Authority), involving SAFRAN Group, ONERA (French Aeronautics and Space Research Center) and CNRS (National Center for Scientific Research).

\section{References}

1. Modi D, Johnson M, Long A, et al. Analysis of pressure profile and flow progression in the vacuum infusion process. Compos Sci Technol 2009; 69: 1458-1464.

2. Kang MK, Lee WI and Hahn HT. Analysis of vacuum bag resin transfer molding process. Compos Part A 2001; 32: $1553-1560$.

3. Williams C, Summerscales J and Grove S. Resin infusion under flexible tooling (RIFT): a review. Compos Part A 1996; 27: 517-524.

4. Han K, Jiang S, Zhang C, et al. Flow modeling and simulation of SCRIMP for composites manufacturing. Compos Part A 2000; 31: 79-86.

5. Celle P, Drapier S and Bergheau J-M. Numerical modelling of liquid infusion into fibrous media undergoing compaction. Eur J Mech-A/Solids 2008; 27: 647-661.

6. Park $\mathrm{CH}$ and Saouab A. Analytical modeling of composite molding by resin infusion with flexible tooling: VARI and RFI processes. J Compos Mater 2009; 43: 1877-1900.

7. Song YS. Mathematical analysis of resin flow through fibrous porous media. Appl Compos Mater 2006; 13: $335-343$. 
8. Lopatnikov S, Simacek P, GillespieJr J, et al. A closed form solution to describe infusion of resin under vacuum in deformable fibrous porous media. Model Simul Mater Sci Eng 2004; 12: S191-S204.

9. Tari MJ, Imbert JP, Lin MY, et al. Analysis of resin transfer molding with high permeability layers. J Manuf Sci Eng 1998; 120: 609-616.

10. Hsiao KT, Mathur R, Advani SG, et al. A closed form solution for flow during the vacuum assisted resin transfer molding process. J Manuf Sci Eng ASME 2000; 122: 463-475.

11. Mathur R, Heider D, Hoffmann C, et al. Flow front measurements and model validation in the vacuum assisted resin transfer molding process. Polym Compos 2001; 22: 477-490.

12. Song YS and Youn JR. Modeling of resin infusion in vacuum assisted resin transfer molding. Polym Compos 2008; 29: 390-395.

13. Trochu F and Gauvin R. Limitations of a boundary fitted finite difference method for the simulation of the resin transfer moulding proccess. $J$ Reinf Plast Compos 1992; 11: 772-786.

14. Acheson JA, Simacek $P$ and Advani SG. The implications of fiber compaction and saturation on fully coupled VARTM simulation. Compos Part A 2004; 35 : 159-169.

15. Kessels JF a, Jonker AS and Akkerman R. Fully flow modeling of resin infusion under flexible tooling using unstructured meshes and wet and dry compaction properties. Compos Part A 2007; 38: 51-60.

16. Gantois R, Cantarel A, Dusserre G, et al. Mold filling simulation of resin transfer molding combining BEM and level set method. Appl Mech Mater 2011; 62: 57-65.

17. Rouhi MS, Wysocki $M$ and Larsson R. Modeling of coupled dual-scale flow-deformation processes in composites manufacturing. Compos Part A 2013; 46: 108-116.

18. Rouhi MS, Wysocki M and Larsson R. Experimental assessment of dual-scale resin flow-deformation in composites processing. Compos Part A 2015; 76: 215-223.

19. Sirtautas J, Pickett AK and Lépicier P. A mesoscopic model for coupled drape-infusion simulation of biaxial non-crimp fabric. Compos Part B 2013; 47: 48-57.

20. Poorzeinolabedin M, Parnas L and Dashatan SH. Resin infusion under flexible tooling process and structural design optimization of the complex composite part. Mater Des 2014; 64: 450-455.

21. Dereims a, Drapier S, Bergheau J-M, et al. 3D robust iterative coupling of Stokes, Darcy and solid mechanics for low permeability media undergoing finite strains. Finite Elem Anal Des 2015; 94: 1-15.

22. (Jonathan) Dong C. An equivalent medium method for the vacuum assisted resin transfer molding process simulation. J Compos Mater 2005; 40: 1193-1213.

23. Calado VM, a and Advani SG. Effective average permeability of multi-layer preforms in resin transfer molding. Compos Sci Technol 1996; 56: 519-531.
24. Chen R, Dong C, Liang Z, et al. Flow modeling and simulation for vacuum assisted resin transfer molding process with the equivalent permeability method. Polym Compos 2004; 25: 146-164.

25. Yoon MK and Dolan DF. Homogenous modeling of VARTM processes with hybrid layered media. J Compos Mater 2008; 42: 805-824.

26. Simacek P and Advani SG. "Squivalent" permeability and flow in compliant porous media. Compos Part $A$ 2016; 80: 107-110.

27. Kang MK and Lee W, Il. A dual-scale analysis of macroscopic resin flow in vacuum assisted resin transfer molding. Polym Compos 2004; 25: 510-520.

28. Yang B, Tang Q, Wang S, et al. Three-dimensional numerical simulation of the filling stage in resin infusion process. J Compos Mater 2016; 50: 4171-4186.

29. Young W-B. A two-layer model for the simulation of the VARTM process with resin distribution layer. Appl Compos Mater 2013; 20: 1305-1319.

30. Ouahbi T, Saouab A, Bréard J, et al. Modelling of hydromechanical coupling in infusion processes. Compos Part A 2007; 38: 1646-1654.

31. Kempner EA and Hahn HT. A unified approach to manufacturing simulation for composites. In: Proceeding of the first Korea-U.S. workshop on composite materials, Seoul, Korea, 1998.

32. Darcy H. Les fontaines publiques de la ville de Dijon. Recherche, Dalmont, Paris. 1856, p.647.

33. Terzaghi K. Theoretical soil mechanics. Géotechnique. New-York: Wiley, 1943, p.510.

34. Kang $\mathrm{M}$ and Lee W, Il. A flow-front refinement technique for the numerical simulation of the resin-transfer molding process. Compos Sci Technol 1999; 59: 1663-1674.

35. Grimsley BW, Cano RJ, Hubert P, et al. Preform characterization in vartm process model development. In: International SAMPE technical conference, San Diego, CA, United States, 2004, pp.1115-1128.

36. Scholz S, Gillespie JW and Heider D. Measurement of transverse permeability using gaseous and liquid flow. Compos Part A 2007; 38: 2034-2040.

37. Um MK, Daniel IM and Childs BW. A gas flow method for determination of in-plane permeability of fiber preforms. Polym Compos 2001; 22: 47-56.

38. Kim SK and Daniel IM. Transient gas flow technique for inspection of fiber preforms in resin transfer molding. Compos Part A 2005; 36: 1694-1699.

39. Kim SK, Opperer JG and Daniel IM. Gas flow method for detecting local preform defects by inverse estimation of space-varying permeability. J Compos Mater 2003; 37 : 1367-1383.

40. Kim SK, Opperer JG and Daniel IM. Determination of permeability of fibrous medium considering inertial effects. Int Commun Heat Mass Transf 2002; 29: 879-885.

41. Laine B. Influence des déformations d'un renfort fibreux sur la perméabilité: modélisations et expériences. $\mathrm{PhD}$ Thesis, Art et Métiers ParisTech, Paris, France, 2008. 
42. Sutton M, Wolters W, Peters W, et al. Determination of displacements using an improved digital correlation method. Image Vis Comput 1983; 1: 133-139.

43. Image J, 1997. Available at: http://imagej.nih.gov/ij/.

44. Xiao X, Hu J, Hua T, et al. Through-thickness air permeability of woven fabric under low pressure compression. Text Res $J$ 2015; 85: 1732-1742.
45. Hirasaki GJ. Flow and transport in porous media I. Geology, chemistry and physics of fluid transport. Chapter 4: volumetric flux: Darcy's law, 2000. Available at: http://www.owlnet.rice.edu/ ceng $571 /$ notes.htm. 